\title{
Virulence and Diversity of Wheat Leaf Rust in the United States in 1993 to 1995
}

\author{
D. L. Long, Plant Pathologist, K. J. Leonard, Research Plant Pathologist, Cereal Disease Laboratory, USDA, Ag- \\ ricultural Research Service, Department of Plant Pathology, University of Minnesota, 1551 Lindig St., St. Paul \\ 55108, and J. J. Roberts, Research Plant Pathologist, USDA-ARS, Georgia Agricultural Experiment Station, Ex- \\ periment 30212
}

\begin{abstract}
Long, D. L., Leonard, K. J., and Roberts, J. J. 1998. Virulence and diversity of wheat leaf rust in the United States in 1993 to 1995. Plant Dis. 82:1391-1400.

Isolates of Puccinia triticina were obtained from wheat leaf collections made by cooperators throughout the United States and from cereal rust field surveys of the Great Plains, Ohio Valley, and Gulf Coast states in 1993, 1994, and 1995. Sixty-two virulence/avirulence phenotypes on 14 host lines that are near-isogenic for leaf rust resistance were found among 681 single uredinial isolates in 1993, 42 phenotypes were found among 683 isolates in 1994, and 51 among 701 isolates in 1995. As in previous surveys, regional race distribution patterns showed that the central United States is a single epidemiological unit distinct from the eastern United States. The distinctive racial composition of collections from the Southeast, Northeast, and Ohio Valley indicates that populations of $P$. triticina in those areas are discrete, suggesting epidemics originate from localized overwintering sources.
\end{abstract}

Additional keywords: plant disease monitoring, Puccinia recondita, rust epidemiology

Wheat leaf rust, caused by Puccinia triticina Erikss. (1) (=P. recondita Desmaz. f. sp. tritici), occurs annually throughout most wheat (Triticum aestivum) growing areas of the United States. In recent years, yield losses to leaf rust have ranged from traces in many states to $11 \%$ in Kansas in 1993, 5\% in California in 1994, and 5\% in Kansas in 1995. Losses to leaf rust in winter wheat in the United States were estimated at $0.9,2.3,3.3,4.8,4.8,0.7$, and $2.4 \%$ in $1989,1990,1991,1992,1993$, 1994, and 1995, respectively (D. L. Long, unpublished). During these years, spring wheat has not suffered significant losses to leaf rust in the United States, mainly because of more effective resistance in spring wheat cultivars and because $P$. triticina rarely overwinters in the northern Great Plains, where most spring wheat is grown.

Wheat leaf rust virulence surveys have been conducted by the Cereal Rust Laboratory, now known as the Cereal Disease Laboratory, since 1978 to detect new pathogenic races and to monitor shifts of

Corresponding author: D. L. Long

E-mail: davidl@puccini.crl.umn.edu

Paper No. 981220035, Scientific Journal Series, Minnesota Agricultural Experiment Station.

Accepted for publication 12 September 1998.

Publication no. D-1998-1027-02R

(C) 1998 The American Phytopathological Society race frequencies in major wheat growing regions of the United States (15). Similar surveys have been done in Canada since 1931 (6) and in Mexico since 1988 (26). In the United States (9) and Canada (5-8), leaf rust survey data have been used to characterize virulence and race dynamics and phenotypic diversity within and between wheat growing regions.

The objectives of this study were to characterize the virulence of $P$. triticina populations in the United States from 1993 through 1995 to the North American wheat leaf rust differentials (10) and other selected lines of wheat, and to compare these results with those of previous surveys.

\section{MATERIALS AND METHODS}

Collections and race identification. Uredinial collections of leaf rust were made from wheat in annual surveys (over routes totaling approximately $22,000 \mathrm{~km}$ ) of the Great Plains, Ohio Valley, and Gulf Coast and by cooperators throughout the United States. From 1993 to 1995, field surveys of wheat were made in southern and central Georgia (late March through May); eastern and southern Texas (early April); northern Texas and south central Oklahoma (late April); southeastern Gulf Coast (mid-April to early May); southeastern states (early May); Oklahoma and Kansas (mid-May); the Ohio River Valley (early June); northwestern Kansas, Nebraska, western Iowa, South Dakota, and southern Minnesota (mid-June); and north- ern Plains states (early July and again in late July). Visual inspections for the presence of rust were made in commercial fields ( 4 to $50 \mathrm{ha}$ ) every $32 \mathrm{~km}$ or in the first field thereafter. Additional collections were made at nurseries and wheat trap plots along the route. Nurseries typically contain a wide array of leaf rust resistance phenotypes, including breeding lines with leaf rust resistance genes not yet in commercial cultivars. Trap plots usually contain older cultivars of wheat no longer prominent in commercial production. Collections were also made from Triticum cylindricum (Aegilops cylindrica) growing near wheat fields in Oklahoma and Texas. A collection consisted of one to several leaves bearing uredinia from a single plant or cultivar. Collections from artificially inoculated nurseries were excluded from the survey.

Urediniospores from each collection were used to inoculate 7-day-old seedlings of the wheat cultivar Thatcher (CI 10003) treated at emergence with maleic hydrazide at approximately $0.01 \mathrm{~g}$ (dissolved in 30 $\mathrm{ml}$ of $\mathrm{H}_{2} \mathrm{O}$ ) per pot to enhance spore production. Plants were sprayed at a rate of approximately $0.5 \mathrm{ml}$ per pot of 10 to 20 seedlings with a suspension of spores in lightweight mineral oil. Inoculated plants were placed in a dew chamber overnight at $18^{\circ} \mathrm{C}$. The plants were then transferred to a greenhouse where temperatures varied between 18 and $28^{\circ} \mathrm{C}$ daily under natural light. After 12 to 15 days, three seedlings were saved per collection, each with the primary leaf trimmed to bear a single uredinium. Six to 9 days later, a cyclone spore collector was used to collect urediniospores separately from one or two such uredinia per collection. If the single uredinia were small and few spores were collected, the isolates were increased through one uredinial generation on Thatcher before inoculating differential lines. Otherwise, spores from the single uredinium were directly inoculated onto 7- to 8-dayold plants of the differential host series (five to seven plants per line) of near isogenic lines of Thatcher with single resistance genes $L r l, 2 a, 2 c, 3,3 k a, 9,10$, $11,16,17,18,24,26$, and 30 (22). Sets of differential lines grown during June through September received no supple- 
mental light. From October through May, natural daylight was supplemented with fluorescent lights from 0200 to 1400 (400 to $450 \mu \mathrm{E} \mathrm{m}^{-2} \mathrm{~s}^{-1}$ at bench level). After 10 to 14 days, infection types were recorded as either high (IT 3 to 4 ) or low (IT 0 to $2+)$ as in previous surveys (12-18).

Race designations and the wheat differentials used to define the races were those agreed upon by the North American Leaf Rust Workers Committee in 1986 (10). Differentials in the set were chosen because they provide distinct differences between low and high infection types and because they account for a large part of the detectable diversity in virulence of $P$. triticina in North America.

Race and virulence frequencies were determined for collections from each of eight agroecological geographic areas (Fig. 1 ): area 1 , mainly southern-adapted soft red winter wheats; areas 2 and 3, mostly northern-adapted soft red and white winter wheat that appears to be epidemiologically separated by geographic features; area 4 , mixed wheat types but primarily hard red winter; area 5 , hard red winter wheat; area 6 , mixed wheat types but primarily hard red spring and durum; area 7 , spring wheats planted in late fall; and area 8, mixed wheat types but primarily soft white winter.

A second sample of spores from each rust collection was bulked with nine other collections made in the same area. Bulked collections were tested on a series of wheat lines resistant to common leaf rust races. This series in 1993 and 1994 consisted of Thatcher near isolines RL6040 (Lr19), RL6043 (Lr21), and RL6080 (Lr29) (22); Aepoglom; Arapahoe (Lr16,24); AZ-FH 50-4-1-1-1 (17); AZ-FH 50-1-1-1-1 (17); Buck Manantial (Lr3, 13, 16, 17, 34) (3); Ceruga 1 and 3 (11); CI 17906 (Lr9, 24) (18); CK 9877; Era/Lr19; Fla 304; Norm; ProBrand 812 (Lr16); RL 6059 (Lr33, 34)

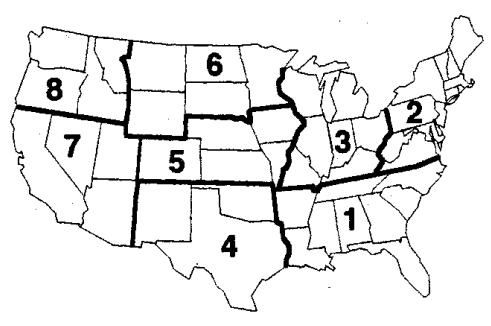

Fig. 1. Agroecological areas for Puccinia triticina in the United States. Area 1, mainly southern-adapted soft red winter wheats; areas 2 and 3, mostly northern-adapted soft red and white winter wheats that appear to be epidemiologically separated by geographic features; area 4, a mixture of wheat types but primarily hard red winter; area 5, hard red winter wheats; area 6 , mixed wheat types, but primarily hard red spring wheat and durum; area 7 , spring wheats planted in late fall; and area 8 , mixed wheat types, but primarily soft white winter types.
(23); E84018 (Lr36); RL 6081 (Lr37); Stoa; TcLr26/TcLr29//TcLr19/3/TcLr26/TcLr21; TcLr29/TcLr21//TcLr25/3/Stoa; Transec (Lr25); and Thatcher, a susceptible check. In 1995, five wheat lines, ProBrand 812, E84018 (Lr36), RL 6081 (Lr37), Ceruga 1, and Stoa, were dropped and replaced by BM/Polk//On/1592, KS86WGRC02 (Lr21 = Lr39) (2), KS86WGRC11 (Lr42) (2), KS86WGRC16 (Lr43) (2), and Jagger. Each entry in the series consisted of five to seven plants. This series was inoculated separately with 56,40 , and 34 bulked collections in 1993, 1994, and 1995, respectively.

Data analysis. The Shannon index (4) of genetic diversity was calculated from data on race frequencies among field and nursery collections in areas 1 through 8 . The Shannon index reflects both the number of races per population and the relative evenness of their frequencies. It is calculated by the equation $H_{w}=-\Sigma p_{i} \log _{e}\left(p_{i}\right)$ in which $p_{i}$ is the frequency of the $i$ th race. Standard errors were calculated, and paired Shannon indexes were compared statistically by the $t$ test as described by Poole (21).

Rogers' index was calculated for all paired comparisons of populations in areas 1 to 8 as previously described (9). Rogers' index measures similarity of racial composition between populations (4). It is calculated by the equation $H_{r}=0.5 \Sigma\left|p_{A i}-p_{B i}\right|$, in which $\left|p_{A i}-p_{B i}\right|$ is the absolute value of the difference between the frequencies of the $i$ th race in populations A and B (4). Rogers' index varies from 0 for paired populations with identical races at identical frequencies to 1.0 for populations with no races in common.

Rogers' index is not sensitive to gene frequencies, because it treats all races as equally distinct, regardless of how many virulences they share. Therefore, a simple similarity index was calculated as a check against Rogers' index. This similarity index, which is the mean of absolute values for differences in virulence frequencies between paired populations, replaces calculations of Nei's (20) standard genetic distance used in previous leaf rust surveys $(9,12,13)$. Because Nei's standard genetic distance is based on similarity of allele frequencies at a number of genetic loci in the respective populations, it may not be appropriate for rust surveys in which virulence frequencies but not allele frequencies are determined. Virulence tests with isolates of $P$. triticina provide phenotypic data but do not reveal whether the phenotypes are heterozygous or homozygous.

Our similarity index for virulence frequencies was calculated according to the formula:

$$
S I=\frac{1}{N} \sum_{i=1}^{N}\left|p_{i A}-p_{i B}\right|
$$

in which $N$ is the number of host lines in the differential set, $p_{i A}$ is the frequency of virulence on the $i$ th differential in popula- tion $\mathrm{A}$, and $p_{i B}$ is the frequency of virulence on the $i$ th differential in population B. Values of SI can vary from 0 for identical populations to 1.0 for pairs of populations with no virulences in common.

\section{RESULTS AND DISCUSSION}

Races identified. In 1993, 1994, and 1995 , totals of 681,683 , and 701 single uredinial isolates, respectively, were characterized. Sixty-two races were identified in 1993, 42 in 1994, and 51 in 1995 (Tables 1 to 4 ) based on high/low infection types on the 12 differential host lines plus Thatcher lines with $\mathrm{LrlO}$ and $\mathrm{Lrl8}$ (10). Races found in areas 1 to 8 are arranged by North American wheat leaf rust code (10) in Tables 2 to 4 and Figure 2. Results are presented as percentages of isolates within areas separated into collections made from nurseries and fields.

The M- race group (virulent on $\mathrm{Lrl}$ and 3) comprised $50 \%$ of the total number of leaf rust isolates identified in 1993, 71\% in 1994 , and $72 \%$ in 1995 . The most frequently identified race in 1993 was MBG10 (virulent on $\operatorname{Lrl}, 3,10$, and 11 ), which comprised $20 \%$ of the nationwide sample. MBG-10 was the found in areas 1, 2, 3, 4, 5 , and 7 of the United States in all 3 years from 1993 to 1995 (Tables 2 to 4, Fig. 2). In 1993, MBG-10 occurred at $>17 \%$ frequency in areas 1,3 , and 4 , where it was the most frequent race (Tables 2 to 4 ). In 1994 and 1995, MBG-10 comprised 16 and $21 \%$, respectively, of the nationwide sample and was the second most frequently identified race. In 1992 (13) and 1991 (12), MBG-10 comprised 17 and 20\%, respectively, of the nationwide sample.

In 1994 and 1995, MBR-10 (virulent on $L r 1,3,3 k a, 10,11$, and 30), the most frequently found race, comprised 36 and $28 \%$ of the nationwide sample, respectively. MBR-10 was found in areas 1 to 7 of the United States in both years, but it was especially common in the Great Plains (areas 4, 5, and 6). Race MBR-10 was not identified in the 1992 survey (13), but in 1993, MBR-10 comprised 6\% of the nationwide sample (Tables 2 to 4), with frequencies of greater than $10 \%$ in areas 5 and 6 (Table 3).

Race group MF- (virulent to $\operatorname{Lr1}, 3,24$, and 26) comprised 9,7 , and $1 \%$ of the nationwide sample in 1993, 1994, and 1995, respectively. Race group MF- was common in areas 4, 5, and 6 in both 1993 and 1994 (Table 3). Thirteen, 11, and 16 of the other M- phenotypes (virulent to $\mathrm{Lrl}$ and 3) comprised 16,12 , and $22 \%$ of the nationwide sample in 1993, 1994, and 1995, respectively (Tables 2 to 4).

Fourteen, seven, and nine T- races (virulent on $\operatorname{Lrl}, 2 a, 2 c$, and 3) formed the second largest group of races, which comprised 31,22 , and $21 \%$ of the nationwide sample in 1993, 1994, and 1995, respectively (Tables 2 to 4 ). The second most frequently identified race in 1993 was TLG-18, which comprised $9 \%$ of the na- 
tionwide sample, primarily because of its high frequency $(26 \%)$ in area 1 (Table 2). In 1994 and 1995, TLG-18 comprised 1 and $6 \%$, respectively, of the nationwide sample. In 1992 (13) and 1991 (12), the TLG-18 race comprised 3 and $1 \%$, respectively, of the nationwide sample. Race TBG-10 was the most common race in 1992 at $21 \%$ of total isolates, but it made up just $5 \%$ of the nationwide sample in $1993,2 \%$ in 1994 , and $2 \%$ in 1995 . Race TDB-10 was the fifth most widely identified race in 1993 and third most widely identified in 1994, when it comprised 6 and $12 \%$ of the nationwide sample, respectively. In 1995, TDB-10 comprised 2\% of the isolates.

The $\mathrm{M}$ - and T- race groups were widely distributed throughout areas 4, 5, and 6 in 1993, 1994, and 1995 (Table 2, Fig. 2), which is consistent with our earlier conclusion that these areas represent a continuous south-north epidemiological unit (9).

From 1981 through 1986 (15-17), the $\mathrm{K}$ - races (virulent on $\operatorname{Lr} 2 a, 2 c$, and 3) were the most frequently identified races nationwide. The frequency of this group has decreased from $39 \%$ in 1985 (16) to $3 \%$ in 1993, 0.1\% in 1994, and none in 1995.

In 1993 to 1995 , most of the P- races (virulent on $L r l, 2 c$, and 3) were found in the eastern soft winter wheat region (areas 1, 2, and 3) and in the Pacific Northwest (area 8) (Tables 2 and 4). Race PNM-10,18 (virulent on $L r l, 2 c, 3,3 k a, 10,18,24$, and 30) comprised $4 \%$ of the nationwide sample in 1993, which was the first year this race was identified. In 1993, $11 \%$ of the isolates from area 1 were of race PNM10,18 . This can be accounted for by the many leaf rust collections made from cv. Coker 9877 ( $\operatorname{Lr} 9$ and 24 resistance), which was grown on a significant acreage throughout area 1 (D. L. Long, unpublished). This was the first time the $\operatorname{Lr} 9$ and 24 gene virulence combination was found in the United States. By 1994 and 1995, the planted acreage of Coker 9877 had decreased significantly, and $\mathrm{PN}$ - races comprised only $1 \%$ of the nationwide sample in both years, and only $4 \%$ and $<1 \%$ in area 1 in 1994 and 1995. In 1994, the PMrace group was found in Pennsylvania. This was the first time the $\operatorname{Lr} 9$ and 26 gene virulence combination was found in the United States.

In the nationwide surveys from 1993 to 1995, the race groups B- (avirulent), C(virulent on $L r 3$ ), and D- (virulent on $L r 2 c$ ) represented less than $2 \%$ of all isolates identified (Tables 2 to 4). In 1993, B- races comprised $1 \%$ of the isolates and were found scattered from Texas to Indiana to North Dakota. The C- races comprised about $1 \%$ of the isolates identified in 1993 , 1994, and 1995, and were scattered from Georgia to California. Most of the D(virulent on $L r 2 c$ ), F- (virulent on $L r 2 c$ and 3), L- (virulent on Lrl), and $\mathrm{N}$ - races (virulent on $\mathrm{Lrl}$ and $2 c$ ) were found in the eastern soft winter wheat region (areas 1, 2, and 3) (Table 2). Races NCB-10, PBP10 , and PGD-10 were unique to area 8 (Table 4). Race NBB-10,18 was found scattered throughout the country, from Virginia and New York to Oregon and Washington.

In 1993 and 1995, the S- race group (virulent to $L r 1,2 a$, and $2 c$ ) was identified from T. cylindricum collections made in southwestern Oklahoma and comprised $<1 \%$ of the total isolates nationwide in 1993 and 1995.

Virulence frequencies. Frequencies of virulence on each of the 14 differential lines differed among collections from the eight agroecological areas (Table 5). Due to variation in greenhouse environment, temperature sensitive genes $L r 3 \mathrm{ka}, 11,17$, and 18 may have been misclassified occasionally. Incidence of virulence on lines with $\operatorname{Lr} 24$ increased from $12 \%$ of the total U.S. isolates in 1991 (12) to $37 \%$ in 1993. Virulence to $\operatorname{Lr} 26$ increased from $1 \%$ in 1986 (17) to $24 \%$ in 1993 . Virulences to Lr24 and $L r 26$ were most common in areas 4, 5, and 6 (Table 5). Cultivars with $\operatorname{Lr} 24$ are widely grown in the southern Great Plains (19). Combined virulence to $\operatorname{Lr} 24$ and $\operatorname{Lr} 26$ was found in KF-, MF-, and TFrace groups in 1993 to 1995 .

Virulence to $\mathrm{Lrll}$ was common throughout the United States and was identified in 24,20 , and 26 virulence combinations in

Table 1. North American wheat leaf rust nomenclature code and corresponding virulence formula for races identified in 1993 to 1995 in the United States

\begin{tabular}{|c|c|c|c|}
\hline Code $^{y}$ & Virulence formula ${ }^{z}$ & Code & Virulence formula \\
\hline BBB-10 & 10 & MFB-10 & $1,3,10,24,26$ \\
\hline BBD-10 & 10,17 & MFC-10 & $1,3,10,24,26,30$ \\
\hline CBB-10 & 3,10 & MFL-10 & $1,3,3 \mathrm{ka}, 10,24,26$ \\
\hline $\mathrm{CBG}$ & 3,11 & MFM-10 & $1,3,3 \mathrm{ka}, 10,24,26,30$ \\
\hline CBG-10,18 & $3,10,11,18$ & MFR-10 & $1,3,3 \mathrm{ka}, 10,11,24,26,30$ \\
\hline CBM-18 & $3,3 k a, 18,30$ & MGB-10 & $1,3,10,16$ \\
\hline CBT-18 & $3,3 \mathrm{ka}, 11,17,18,30$ & MGD-10 & $1,3,10,16,17$ \\
\hline CCB-10 & $3,10,26$ & MGG-10 & $1,3,10,11,16$ \\
\hline CCM-18 & $3,3 \mathrm{ka}, 18,26,30$ & MJB-10 & $1,3,10,16,24$ \\
\hline CCR-10,18 & $3,3 \mathrm{ka}, 10,11,18,26,30$ & NBB-10,18 & $1,2 c, 10,18$ \\
\hline CGB-10 & $3,10,16$ & NCB-10 & $1,2 c, 10,26$ \\
\hline DBB-18 & $2 c, 18$ & PBB-10 & $1,2 c, 3,10$ \\
\hline FBB-10 & $2 c, 3,10$ & PBB-10,18 & $1,2 c, 3,10,18$ \\
\hline FBM & $2 c, 3,3 k a, 30$ & PBD-10 & $1,2 c, 3,10,17$ \\
\hline FBM-18 & $2 c, 3,3 k a, 18,30$ & PBG-10 & $1,2 c, 3,10,11$ \\
\hline FBR-10,18 & $2 c, 3,3 k a, 10,11,18,30$ & PBJ-10,18 & $1,2 c, 3,10,11,17,18$ \\
\hline FBR-18 & $2 c, 3,3 k a, 11,18,30$ & PBL-10 & $1,2 c, 3,3 \mathrm{ka}, 10$ \\
\hline FCD-10 & $2 c, 3,10,17,26$ & PBM-18 & $1,2 \mathrm{c}, 3,3 \mathrm{ka}, 18,30$ \\
\hline FCR-18 & $2 c, 3,3 k a, 11,18,26,30$ & PBM-10,18 & $1,2 \mathrm{c}, 3,3 \mathrm{ka}, 10,18,30$ \\
\hline FMB-18 & $2 c, 3,9,18,26$ & PBP-10 & $1,2 \mathrm{c}, 3,3 \mathrm{ka}, 10,17,30$ \\
\hline KBG-10 & $2 a, 2 c, 3,10,11$ & PBQ-10,18 & $1,2 \mathrm{c}, 3,3 \mathrm{ka}, 10,11,18$ \\
\hline KCG-10 & $2 a, 2 c, 3,10,11,26$ & PBR-10,18 & $1,2 \mathrm{c}, 3,3 \mathrm{ka}, 10,11,18,30$ \\
\hline KDB-10 & $2 a, 2 c, 3,10,24$ & PGD-10 & $1,2 c, 3,10,16,17$ \\
\hline KFB-10 & $2 a, 2 c, 3,10,24,26$ & PLM-18 & $1,2 c, 3,3 k a, 9,18,30$ \\
\hline LBB-10,18 & $1,10,18$ & PLM-10,18 & $1,2 c, 3,3 \mathrm{ka}, 9,10,18,30$ \\
\hline LBG-10 & $1,10,11$ & PLR-10,18 & $1,2 c, 3,3 k a, 9,10,11,18,30$ \\
\hline LBG-10,18 & $1,10,11,18$ & PMM-10,18 & $1,2 c, 3,3 k a, 9,10,18,26,30$ \\
\hline LCB-10,18 & $1,10,18,26$ & PMR-10,18 & $1,2 \mathrm{c}, 3,3 \mathrm{ka}, 9,10,11,18,26,30$ \\
\hline LCG-10,18 & $1,10,11,18,26$ & PNM-10 & $1,2 c, 3,3 k a, 9,10,24,30$ \\
\hline MBB & 1,3 & PNM-10,18 & $1,2 c, 3,3 \mathrm{ka}, 9,10,18,24,30$ \\
\hline MBB-10 & $1,3,10$ & PNR-10,18 & $1,2 \mathrm{c}, 3,3 \mathrm{ka}, 9,10,11,18,24,30$ \\
\hline MBB-18 & $1,3,18$ & SBB-10 & $1,2 a, 2 c, 10$ \\
\hline MBB-10,18 & $1,3,10,18$ & SBD & $1,2 a, 2 c, 17$ \\
\hline MBD-10 & $1,3,10,24$ & SBJ & $1,2 a, 2 c, 11,17$ \\
\hline MBG & $1,3,11$ & TBB-10 & $1,2 a, 2 c, 3,10$ \\
\hline MBG-10 & $1,3,10,11$ & TBD-10 & $1,2 a, 2 c, 3,10,17$ \\
\hline MBG-18 & $1,3,11,18$ & TBG & $1,2 a, 2 c, 3,11$ \\
\hline MBG-10,18 & $1,3,10,11,18$ & TBG-18 & $1,2 a, 2 c, 3,11,18$ \\
\hline MBJ-10 & $1,3,10,11,17$ & TBG-10 & $1,2 a, 2 c, 3,10,11$ \\
\hline MBM-10 & $1,3,3 \mathrm{ka}, 10,30$ & TBG-10,18 & $1,2 a, 2 c, 3,10,11,18$ \\
\hline MBR & $1,3,3 \mathrm{ka}, 11,30$ & TBJ-10,18 & $1,2 a, 2 c, 3,10,11,17,18$ \\
\hline MBR-10 & $1,3,3 \mathrm{ka}, 10,11,30$ & TBR-10 & $1,2 a, 2 c, 3,3 k a, 10,11,30$ \\
\hline MBR-10,18 & $1,3,3 \mathrm{ka}, 10,11,18,30$ & TBT & $1,2 a, 2 c, 3,3 k a, 11,17,30$ \\
\hline MCB-10 & $1,3,10,26$ & TCG-10 & $1,2 a, 2 c, 3,10,11,26$ \\
\hline MCD-10 & $1,3,10,17,26$ & TDB-10 & $1,2 a, 2 c, 3,10,24$ \\
\hline MCG-10 & $1,3,10,11,26$ & TDG-10 & $1,2 a, 2 c, 3,10,11,24$ \\
\hline MCG-10,18 & $1,3,10,11,18,26$ & TDJ-10,18 & $1,2 a, 2 c, 3,10,11,17,18,24$ \\
\hline MCR-10 & $1,3,3 \mathrm{ka}, 10,11,26,30$ & TDR-10 & $1,2 a, 2 c, 3,3 \mathrm{ka}, 10,11,24,30$ \\
\hline MDB-10 & $1,3,10,24$ & TFB-10 & $1,2 a, 2 c, 3,10,24,26$ \\
\hline MDG-10 & $1,3,10,11,24$ & TFG-10 & $1,2 a, 2 c, 3,10,11,24,26$ \\
\hline MDR-10 & $1,3,3 \mathrm{ka}, 10,11,24,30$ & TLG-18 & $1,2 a, 2 c, 3,9,11,18$ \\
\hline
\end{tabular}

y Race code plus $L r 10$ and $\operatorname{Lr} 18$ near-isogenic supplements.

${ }^{\mathrm{z}}$ Resistances evaluated: $\mathrm{Lrl}, 2 \mathrm{a}, 2 \mathrm{c}, 3,9,16,24,26,3 \mathrm{ka}, 11,17,30,10$, and 18 . 
Table 2. Races of Puccinia triticina collected from fields (F) and nurseries (N) in agroecological areas 1 (Southeast), 2 (Northeast), and 3 (Ohio Valley) of the United States in 1993, 1994, and 1995

\begin{tabular}{|c|c|c|c|c|c|c|c|c|c|c|c|c|c|c|c|c|c|c|}
\hline \multirow[b]{4}{*}{ Code $^{y}$} & \multicolumn{18}{|c|}{ Percentage of isolates from indicated source } \\
\hline & \multicolumn{6}{|c|}{ Area $1^{\mathbf{z}}$} & & & & & & & & & & & & \\
\hline & & & & 94 & & & & & & & & & & & & & & \\
\hline & $\mathbf{F}$ & $\mathbf{N}$ & $\mathbf{F}$ & $\mathbf{N}$ & $\mathbf{F}$ & $\mathbf{N}$ & $\mathbf{F}$ & $\mathbf{N}$ & $\mathbf{F}$ & $\mathbf{N}$ & $\mathbf{F}$ & $\mathbf{N}$ & $\mathbf{F}$ & $\mathbf{N}$ & $\mathbf{F}$ & $\mathbf{N}$ & $\mathbf{F}$ & $\mathbf{N}$ \\
\hline BBD-10 & 0 & 0 & 0 & 0 & 0 & 0 & 0 & 0 & 0 & 0 & 0 & 0 & 3 & 0 & 0 & 0 & 0 & 0 \\
\hline CBG & 0 & 1 & 0 & 0 & 0 & 0 & 0 & 0 & 0 & 0 & 0 & 0 & 0 & 0 & 0 & 0 & 0 & 0 \\
\hline CBG-10,18 & 0 & 0 & 0 & 0 & 0 & 0 & 0 & 0 & 0 & 0 & 0 & 0 & 0 & 0 & 10 & 0 & 0 & 0 \\
\hline CBM-18 & 0 & 0 & 0 & 0 & 0 & 0 & 0 & 0 & 0 & 0 & 0 & 0 & 3 & 0 & 0 & 0 & 0 & 4 \\
\hline CBT-18 & 0 & 0 & 0 & 0 & 1 & 0 & 0 & 0 & 0 & 0 & 0 & 0 & 0 & 0 & 0 & 0 & 0 & 0 \\
\hline CCB-10 & 0 & 0 & 0 & 0 & 0 & 0 & 0 & 0 & 0 & 0 & 0 & 0 & 0 & 0 & 10 & 0 & 0 & 0 \\
\hline CCM-18 & 0 & 0 & 0 & 0 & 0 & 0 & 0 & 0 & 0 & 0 & 0 & 0 & 0 & 0 & 0 & 0 & 3 & 8 \\
\hline CCR-10,18 & 0 & 0 & 0 & 0 & 0 & 0 & 0 & 0 & 0 & 0 & 0 & 0 & 0 & 0 & 0 & 0 & 0 & 8 \\
\hline DBB-18 & 0 & 0 & 0 & 0 & 0 & 0 & 0 & 10 & 0 & 0 & 0 & 0 & 0 & 0 & 0 & 0 & 0 & 0 \\
\hline FBM & 0 & 0 & 0 & 0 & 0 & 0 & 0 & 0 & 0 & 0 & 0 & 0 & 17 & 0 & 0 & 0 & 0 & 0 \\
\hline FBM-18 & 5 & 1 & 0 & 0 & 0 & 0 & 0 & 0 & 0 & 0 & 0 & 0 & 24 & 0 & 0 & 0 & 0 & 0 \\
\hline FBR-18 & 0 & 0 & 0 & 0 & 0 & 0 & 0 & 0 & 0 & 0 & 0 & 0 & 0 & 0 & 5 & 0 & 0 & 0 \\
\hline FBR-10,18 & 0 & 0 & 0 & 1 & 0 & 0 & 0 & 0 & 0 & 0 & 0 & 0 & 0 & 0 & 0 & 0 & 0 & 0 \\
\hline FCR-18 & 0 & 0 & 0 & 0 & 0 & 0 & 0 & 0 & 0 & 0 & 0 & 0 & 10 & 0 & 0 & 0 & 0 & 0 \\
\hline FMB-18 & 0 & 0 & 0 & 0 & 0 & 0 & 0 & 0 & 0 & 0 & 0 & 0 & 0 & 0 & 0 & 33 & 0 & 0 \\
\hline KBG-10 & 0 & 1 & 0 & 0 & 0 & 0 & 0 & 0 & 0 & 0 & 0 & 0 & 0 & 1 & 0 & 0 & 0 & 0 \\
\hline LBB-10,18 & 0 & 0 & 0 & 0 & 0 & 0 & 0 & 10 & 0 & 0 & 0 & 17 & 0 & 0 & 0 & 0 & 0 & 0 \\
\hline LBG-10,18 & 0 & 0 & 0 & 0 & 0 & 2 & 0 & 0 & 0 & 0 & 0 & 0 & 0 & 0 & 0 & 0 & 0 & 0 \\
\hline LCB-10,18 & 0 & 1 & 0 & 0 & 0 & 3 & 0 & 0 & 0 & 0 & 0 & 0 & 0 & 0 & 0 & 0 & 0 & 0 \\
\hline LCG-10,18 & 0 & 0 & 0 & 0 & 3 & 1 & 0 & 0 & 0 & 0 & 0 & 0 & 0 & 0 & 0 & 0 & 0 & 0 \\
\hline MBB & 0 & 0 & 0 & 0 & 0 & 0 & 0 & 0 & 0 & 0 & 0 & 0 & 0 & 1 & 0 & 0 & 0 & 0 \\
\hline MBB-18 & 0 & 0 & 0 & 0 & 0 & 0 & 0 & 0 & 0 & 0 & 0 & 0 & 0 & 0 & 0 & 0 & 3 & 0 \\
\hline MBB-10 & 0 & 0 & 11 & 3 & 0 & 0 & 0 & 0 & 0 & 0 & 0 & 0 & 0 & 2 & 0 & 0 & 0 & 8 \\
\hline MBG & 4 & 6 & 0 & 0 & 9 & 2 & 0 & 0 & 0 & 8 & 0 & 0 & 3 & 2 & 0 & 4 & 0 & 0 \\
\hline MBG-18 & 0 & 1 & 0 & 0 & 0 & 0 & 0 & 0 & 0 & 0 & 0 & 0 & 0 & 0 & 0 & 0 & 0 & 0 \\
\hline MBG-10 & 43 & 32 & 37 & 40 & 29 & 47 & 0 & 10 & 0 & 25 & 50 & 25 & 14 & 27 & 5 & 17 & 28 & 8 \\
\hline MBG-10,18 & 0 & 0 & 0 & 0 & 8 & 3 & 0 & 0 & 0 & 0 & 0 & 0 & 0 & 0 & 0 & 0 & 0 & 0 \\
\hline MBR & 0 & 0 & 0 & 2 & 0 & 0 & 0 & 0 & 0 & 0 & 0 & 0 & 0 & 0 & 10 & 8 & 0 & 0 \\
\hline MBR-10 & 4 & 2 & 7 & 30 & 22 & 16 & 0 & 0 & 0 & 25 & 0 & 0 & 0 & 10 & 25 & 21 & 34 & 31 \\
\hline MBR-10,18 & 0 & 0 & 0 & 0 & 0 & 3 & 0 & 0 & 0 & 0 & 0 & 0 & 0 & 0 & 0 & 0 & 0 & 0 \\
\hline MCB-10 & 0 & 0 & 0 & 3 & 0 & 0 & 0 & 0 & 0 & 0 & 0 & 0 & 0 & 1 & 0 & 0 & 0 & 0 \\
\hline MCG-10 & 0 & 0 & 0 & 0 & 0 & 0 & 0 & 0 & 0 & 0 & 0 & 8 & 0 & 0 & 0 & 0 & 0 & 0 \\
\hline MCG-10,18 & 0 & 0 & 0 & 0 & 0 & 0 & 0 & 0 & 0 & 0 & 0 & 8 & 0 & 0 & 0 & 0 & 6 & 0 \\
\hline MCR-10 & 0 & 0 & 0 & 0 & 4 & 2 & 0 & 0 & 0 & 0 & 0 & 0 & 0 & 0 & 0 & 0 & 0 & 19 \\
\hline MDB-10 & 0 & 1 & 0 & 0 & 0 & 0 & 0 & 0 & 0 & 0 & 0 & 0 & 0 & 5 & 0 & 0 & 13 & 0 \\
\hline MDG-10 & 0 & 0 & 0 & 0 & 0 & 0 & 0 & 0 & 0 & 0 & 0 & 0 & 0 & 2 & 0 & 0 & 0 & 0 \\
\hline MFB-10 & 5 & 1 & 0 & 2 & 0 & 0 & 0 & 0 & 0 & 0 & 0 & 0 & 0 & 6 & 0 & 0 & 0 & 0 \\
\hline MFC-10 & 0 & 0 & 0 & 0 & 0 & 0 & 0 & 0 & 0 & 0 & 0 & 0 & 0 & 0 & 10 & 0 & 0 & 0 \\
\hline MFL-10 & 0 & 0 & 0 & 0 & 0 & 0 & 0 & 0 & 0 & 0 & 0 & 0 & 0 & 0 & 10 & 0 & 0 & 4 \\
\hline MFM-10 & 0 & 0 & 0 & 0 & 0 & 0 & 0 & 0 & 0 & 0 & 0 & 0 & 7 & 2 & 0 & 0 & 0 & 0 \\
\hline MGB-10 & 0 & 0 & 0 & 0 & 0 & 0 & 0 & 0 & 0 & 0 & 0 & 0 & 0 & 2 & 0 & 0 & 0 & 0 \\
\hline MJB-10 & 0 & 0 & 0 & 0 & 0 & 0 & 0 & 0 & 0 & 0 & 0 & 17 & 0 & 0 & 0 & 0 & 0 & 0 \\
\hline NBB-10,18 & 0 & 0 & 0 & 0 & 0 & 0 & 0 & 40 & 0 & 0 & 0 & 0 & 0 & 0 & 0 & 0 & 0 & 0 \\
\hline PBB-10 & 0 & 0 & 0 & 0 & 0 & 0 & 0 & 0 & 0 & 0 & 0 & 0 & 7 & 0 & 0 & 0 & 0 & 0 \\
\hline PBD-10 & 0 & 1 & 0 & 0 & 0 & 0 & 0 & 0 & 0 & 0 & 0 & 0 & 0 & 0 & 0 & 0 & 0 & 0 \\
\hline PBL-10 & 0 & 0 & 0 & 0 & 0 & 0 & 0 & 30 & 0 & 0 & 0 & 8 & 0 & 0 & 0 & 0 & 0 & 0 \\
\hline PBM-18 & 0 & 0 & 0 & 0 & 0 & 0 & 0 & 0 & 0 & 0 & 0 & 0 & 3 & 0 & 0 & 0 & 0 & 0 \\
\hline PBM-10,18 & 0 & 0 & 0 & 0 & 0 & 0 & 0 & 0 & 0 & 8 & 0 & 0 & 0 & 0 & 0 & 0 & 0 & 0 \\
\hline PBQ-10,18 & 0 & 0 & 0 & 0 & 0 & 0 & 0 & 0 & 0 & 0 & 0 & 0 & 0 & 1 & 0 & 0 & 0 & 0 \\
\hline PBR-10,18 & 0 & 0 & 7 & 0 & 0 & 1 & 0 & 0 & 33 & 17 & 0 & 0 & 0 & 0 & 0 & 0 & 0 & 0 \\
\hline PLM-18 & 0 & 0 & 0 & 0 & 0 & 0 & 0 & 0 & 0 & 0 & 0 & 0 & 0 & 0 & 0 & 0 & 0 & 4 \\
\hline PLM-10,18 & 0 & 0 & 4 & 0 & 0 & 0 & 0 & 0 & 0 & 0 & 0 & 0 & 0 & 0 & 0 & 0 & 0 & 0 \\
\hline PLR-10,18 & 3 & 0 & 0 & 0 & 0 & 0 & 0 & 0 & 0 & 0 & 0 & 0 & 0 & 0 & 0 & 0 & 0 & 0 \\
\hline PMM-10,18 & 0 & 0 & 0 & 0 & 0 & 0 & 0 & 0 & 33 & 0 & 0 & 0 & 0 & 0 & 0 & 0 & 0 & 0 \\
\hline PMR-10,18 & 0 & 0 & 0 & 0 & 0 & 0 & 0 & 0 & 17 & 0 & 0 & 0 & 0 & 0 & 0 & 0 & 0 & 0 \\
\hline PNM-10 & 0 & 0 & 0 & 0 & 0 & 1 & 0 & 0 & 0 & 0 & 0 & 0 & 0 & 0 & 0 & 0 & 0 & 0 \\
\hline PNM-10,18 & 15 & 9 & 4 & 2 & 0 & 1 & 0 & 0 & 0 & 0 & 50 & 0 & 3 & 1 & 10 & 0 & 0 & 0 \\
\hline PNR-10,18 & 0 & 0 & 0 & 0 & 0 & 0 & 0 & 0 & 0 & 0 & 0 & 0 & 0 & 0 & 0 & 0 & 0 & 8 \\
\hline TBG & 1 & 0 & 0 & 0 & 0 & 0 & 0 & 0 & 0 & 0 & 0 & 0 & 0 & 0 & 0 & 0 & 0 & 0 \\
\hline TBG-18 & 0 & 2 & 0 & 0 & 0 & 0 & 0 & 0 & 0 & 0 & 0 & 0 & 0 & 0 & 0 & 0 & 0 & 0 \\
\hline TBG-10 & 0 & 6 & 0 & 2 & 1 & 1 & 0 & 0 & 17 & 8 & 0 & 0 & 0 & 12 & 0 & 0 & 0 & 0 \\
\hline TBG-10,18 & 0 & 0 & 0 & 0 & 1 & 1 & 0 & 0 & 0 & 0 & 0 & 0 & 0 & 0 & 0 & 0 & 0 & 0 \\
\hline TBJ-10,18 & 1 & 1 & 0 & 0 & 0 & 0 & 0 & 0 & 0 & 0 & 0 & 0 & 0 & 0 & 0 & 0 & 0 & 0 \\
\hline TDB-10 & 0 & 1 & 7 & 7 & 0 & 0 & 0 & 0 & 0 & 8 & 0 & 0 & 0 & 6 & 0 & 0 & 0 & 0 \\
\hline TDG-10 & 0 & 0 & 7 & 3 & 0 & 1 & 0 & 0 & 0 & 0 & 0 & 0 & 0 & 0 & 0 & 17 & 0 & 0 \\
\hline TFB-10 & 0 & 0 & 0 & 0 & 0 & 1 & 0 & 0 & 0 & 0 & 0 & 0 & 0 & 15 & 0 & 0 & 3 & 0 \\
\hline TFG-10 & 0 & 2 & 0 & 1 & 4 & 0 & 0 & 0 & 0 & 0 & 0 & 0 & 3 & 1 & 0 & 0 & 9 & 0 \\
\hline TLG-18 & 18 & 30 & 15 & 3 & 18 & 15 & 0 & 0 & 0 & 0 & 0 & 17 & 0 & 0 & 5 & 0 & 0 & 0 \\
\hline Isolates (no.) & 74 & 140 & 27 & 125 & 78 & 159 & 0 & 10 & 6 & 12 & 4 & 12 & 29 & 82 & 20 & 24 & 32 & 26 \\
\hline
\end{tabular}

y Races based on 12 North American leaf rust differential lines (10) plus supplemental lines with $\operatorname{Lr} 10$ and $\operatorname{Lr} 18$.

${ }^{\mathrm{z}}$ Agroecological areas are shown in Figure 1. 
1993, 1994, and 1995, respectively. In the 5 years before 1991, virulence combinations with $\mathrm{Lr} 11$ averaged 13 per year. This reflects a selective advantage of this virulence due to an increase in the acreage of cultivars with Lrll (25). The increase in numbers of races found with virulence to $\mathrm{Lrll}$ also suggests an accumulation of independent mutations to Lrll virulence in a variety of genetic lineages in the $P$. triticina populations. Virulence to $\mathrm{Lrll}$ was most frequent in $\mathrm{M}$ - and T- races, the two most common race groups, as well as in the $\mathrm{P}$ - race group, which occurred both in the eastern soft red winter wheat region (areas 1, 2, and 3) and in the Pacific Northwest (area $8)$.

Virulence to $\operatorname{Lr} 9$ was identified in 13,3 , and $8 \%$ (Table 5) of the nationwide sample of P. triticina in 1993, 1994, and 1995, respectively. In the last 3 years, $L r 9$ virulence has been found only in the PL-, PM-, and $\mathrm{PN}$ - races, mainly in the eastern soft

Table 3. Races of Puccinia triticina collected from fields (F) and nurseries (N) in agroecological areas 4 (Southern Plains), 5 (Cental Plains), and 6 (Northern Plains) of the United States in 1993, 1994, and 1995

\begin{tabular}{|c|c|c|c|c|c|c|c|c|c|c|c|c|c|c|c|c|c|c|}
\hline \multirow[b]{4}{*}{ Code $^{y}$} & \multicolumn{18}{|c|}{ Percentage of isolates from indicated source } \\
\hline & \multicolumn{6}{|c|}{ Area $4^{z}$} & \multicolumn{6}{|c|}{ Area 5} & \multicolumn{6}{|c|}{ Area 6} \\
\hline & \multicolumn{2}{|c|}{1993} & \multicolumn{2}{|c|}{1994} & \multicolumn{2}{|c|}{1995} & \multicolumn{2}{|c|}{1993} & & & & & & & & & & \\
\hline & $\mathbf{F}$ & $\mathbf{N}$ & $\mathbf{F}$ & $\mathbf{N}$ & $\mathbf{F}$ & $\mathbf{N}$ & $\mathbf{F}$ & $\mathbf{N}$ & $\mathbf{F}$ & $\mathbf{N}$ & $\mathbf{F}$ & $\mathbf{N}$ & $\mathbf{F}$ & $\mathbf{N}$ & $\mathbf{F}$ & $\mathbf{N}$ & $\mathbf{F}$ & $\mathbf{N}$ \\
\hline BBB-10 & 1 & 0 & 0 & 0 & 0 & 0 & 0 & 0 & 0 & 0 & 0 & 0 & 0 & 0 & 0 & 0 & 0 & 0 \\
\hline BBD-10 & 0 & 0 & 0 & 0 & 0 & 0 & 0 & 0 & 0 & 0 & 0 & 0 & 0 & 4 & 0 & 0 & 0 & 0 \\
\hline CBB-10 & 3 & 0 & 0 & 0 & 0 & 0 & 0 & 0 & 0 & 0 & 0 & 0 & 0 & 0 & 0 & 0 & 0 & 0 \\
\hline CCB-10 & 1 & 3 & 0 & 0 & 0 & 0 & 4 & 0 & 0 & 0 & 0 & 0 & 0 & 0 & 0 & 0 & 0 & 0 \\
\hline CGB-10 & 0 & 0 & 0 & 0 & 0 & 0 & 0 & 0 & 0 & 0 & 0 & 4 & 0 & 0 & 0 & 0 & 0 & 0 \\
\hline FBB-10 & 1 & 0 & 0 & 0 & 0 & 0 & 0 & 0 & 0 & 0 & 0 & 0 & 0 & 0 & 0 & 0 & 0 & 0 \\
\hline FBM-18 & 1 & 0 & 0 & 0 & 0 & 0 & 0 & 0 & 0 & 0 & 0 & 0 & 0 & 0 & 0 & 0 & 0 & 0 \\
\hline FCD-10 & 0 & 0 & 0 & 0 & 0 & 3 & 0 & 0 & 0 & 0 & 0 & 0 & 0 & 0 & 0 & 0 & 0 & 0 \\
\hline KBG-10 & 1 & 0 & 0 & 0 & 0 & 0 & 8 & 0 & 0 & 0 & 0 & 0 & 0 & 0 & 0 & 0 & 0 & 0 \\
\hline KCG-10 & 0 & 0 & 0 & 0 & 0 & 0 & 0 & 2 & 0 & 0 & 0 & 0 & 0 & 1 & 0 & 0 & 0 & 0 \\
\hline KDB-10 & 0 & 0 & 0 & 0 & 0 & 0 & 0 & 3 & 0 & 0 & 0 & 0 & 0 & 0 & 0 & 0 & 0 & 0 \\
\hline KFB-10 & 1 & 3 & 0 & 1 & 0 & 0 & 0 & 2 & 0 & 0 & 0 & 0 & 0 & 5 & 0 & 0 & 0 & 0 \\
\hline MBB-10 & 0 & 3 & 0 & 0 & 0 & 0 & 0 & 2 & 0 & 0 & 0 & 0 & 0 & 0 & 0 & 3 & 0 & 0 \\
\hline MBB-10,18 & 0 & 0 & 0 & 0 & 0 & 0 & 0 & 0 & 0 & 0 & 0 & 0 & 0 & 0 & 0 & 0 & 0 & 0 \\
\hline MBD-10 & 0 & 0 & 0 & 0 & 0 & 0 & 0 & 2 & 0 & 3 & 0 & 0 & 11 & 1 & 0 & 0 & 0 & 0 \\
\hline MBG & 0 & 0 & 0 & 0 & 0 & 2 & 0 & 0 & 0 & 0 & 0 & 0 & 0 & 0 & 0 & 0 & 0 & 0 \\
\hline MBG-10 & 17 & 18 & 13 & 8 & 7 & 5 & 12 & 2 & 0 & 6 & 13 & 4 & 0 & 10 & 9 & 5 & 0 & 0 \\
\hline MBJ-10 & 0 & 0 & 0 & 0 & 3 & 1 & 0 & 0 & 0 & 0 & 0 & 0 & 0 & 0 & 0 & 0 & 0 & 0 \\
\hline MBM-10 & 0 & 0 & 0 & 0 & 0 & 0 & 0 & 0 & 0 & 0 & 0 & 0 & 0 & 3 & 0 & 0 & 0 & 0 \\
\hline MBR-10 & 8 & 9 & 33 & 32 & 33 & 29 & 0 & 15 & 67 & 63 & 65 & 65 & 11 & 12 & 50 & 52 & 11 & 50 \\
\hline MCB-10 & 0 & 3 & 0 & 0 & 1 & 1 & 8 & 0 & 0 & 0 & 0 & 0 & 0 & 3 & 0 & 0 & 0 & 0 \\
\hline MCD-10 & 0 & 0 & 0 & 2 & 0 & 2 & 0 & 0 & 0 & 0 & 0 & 0 & 0 & 0 & 0 & 0 & 0 & 0 \\
\hline MCG-10 & 3 & 0 & 0 & 0 & 0 & 0 & 8 & 0 & 0 & 0 & 0 & 0 & 0 & 0 & 0 & 0 & 0 & 0 \\
\hline MCR-10 & 0 & 0 & 0 & 0 & 3 & 2 & 0 & 3 & 0 & 0 & 0 & 0 & 11 & 0 & 0 & 1 & 0 & 0 \\
\hline MDB-10 & 7 & 3 & 3 & 5 & 0 & 3 & 0 & 3 & 11 & 0 & 0 & 2 & 11 & 5 & 0 & 3 & 22 & 3 \\
\hline MDG-10 & 0 & 0 & 0 & 0 & 0 & 1 & 0 & 0 & 0 & 0 & 0 & 9 & 0 & 0 & 0 & 0 & 0 & 0 \\
\hline MDR-10 & 1 & 0 & 0 & 0 & 9 & 14 & 4 & 0 & 0 & 0 & 0 & 4 & 0 & 0 & 4 & 3 & 11 & 3 \\
\hline MFB-10 & 17 & 6 & 7 & 12 & 0 & 1 & 15 & 14 & 0 & 3 & 0 & 4 & 22 & 19 & 11 & 9 & 0 & 5 \\
\hline MFM-10 & 0 & 0 & 0 & 0 & 3 & 0 & 0 & 5 & 0 & 0 & 0 & 0 & 0 & 0 & 0 & 0 & 0 & 0 \\
\hline MFR-10 & 0 & 0 & 3 & 0 & 0 & 0 & 0 & 0 & 0 & 0 & 0 & 0 & 0 & 0 & 0 & 5 & 0 & 0 \\
\hline MGB-10 & 0 & 0 & 0 & 0 & 0 & 3 & 0 & 0 & 0 & 0 & 0 & 0 & 0 & 0 & 0 & 0 & 0 & 0 \\
\hline MGD-10 & 0 & 0 & 3 & 0 & 0 & 0 & 0 & 0 & 0 & 0 & 0 & 0 & 0 & 0 & 0 & 0 & 0 & 0 \\
\hline MGG-10 & 0 & 0 & 0 & 0 & 0 & 0 & 0 & 0 & 0 & 0 & 0 & 2 & 0 & 0 & 0 & 0 & 0 & 0 \\
\hline MJB-10 & 0 & 0 & 0 & 0 & 3 & 0 & 0 & 0 & 0 & 0 & 0 & 4 & 0 & 0 & 0 & 0 & 0 & 3 \\
\hline PBB-10,18 & 0 & 0 & 0 & 0 & 0 & 0 & 0 & 0 & 0 & 0 & 0 & 0 & 0 & 0 & 0 & 1 & 0 & 0 \\
\hline PBD-10 & 0 & 0 & 1 & 0 & 0 & 0 & 0 & 0 & 0 & 0 & 0 & 0 & 0 & 0 & 0 & 0 & 0 & 0 \\
\hline PLM-10,18 & 0 & 6 & 0 & 0 & 0 & 0 & 0 & 0 & 0 & 0 & 0 & 0 & 0 & 0 & 0 & 0 & 0 & 0 \\
\hline PNM-10,18 & 0 & 0 & 0 & 0 & 0 & 0 & 0 & 0 & 0 & 0 & 0 & 0 & 0 & 1 & 0 & 0 & 0 & 0 \\
\hline PNR-10,18 & 0 & 0 & 0 & 0 & 3 & 0 & 0 & 0 & 0 & 0 & 0 & 0 & 0 & 0 & 0 & 0 & 0 & 0 \\
\hline SBB-10 & 1 & 6 & 0 & 0 & 0 & 0 & 0 & 0 & 0 & 0 & 0 & 0 & 0 & 0 & 0 & 0 & 0 & 0 \\
\hline SBD & 1 & 0 & 6 & 0 & 6 & 0 & 0 & 0 & 0 & 0 & 0 & 0 & 0 & 0 & 0 & 0 & 0 & 0 \\
\hline SBJ & 0 & 0 & 3 & 0 & 0 & 0 & 0 & 0 & 0 & 0 & 0 & 0 & 0 & 0 & 0 & 0 & 0 & 0 \\
\hline TBB-10 & 1 & 0 & 0 & 0 & 0 & 0 & 0 & 0 & 0 & 0 & 0 & 0 & 0 & 0 & 0 & 0 & 0 & 0 \\
\hline TBD-10 & 3 & 3 & 0 & 2 & 0 & 0 & 0 & 0 & 0 & 0 & 0 & 0 & 0 & 1 & 0 & 1 & 0 & 0 \\
\hline TBG & 0 & 0 & 0 & 0 & 0 & 0 & 4 & 0 & 0 & 0 & 0 & 0 & 0 & 0 & 0 & 0 & 0 & 0 \\
\hline TBG-10 & 3 & 6 & 3 & 1 & 0 & 4 & 8 & 5 & 0 & 4 & 4 & 0 & 11 & 5 & 2 & 3 & 22 & 3 \\
\hline TBR-10 & 0 & 0 & 0 & 0 & 0 & 0 & 0 & 0 & 0 & 0 & 0 & 0 & 0 & 0 & 0 & 0 & 11 & 0 \\
\hline TBT & 0 & 0 & 0 & 0 & 0 & 0 & 0 & 2 & 0 & 0 & 0 & 0 & 0 & 0 & 0 & 0 & 0 & 0 \\
\hline TCG-10 & 0 & 0 & 0 & 0 & 0 & 0 & 0 & 0 & 0 & 0 & 0 & 0 & 0 & 3 & 0 & 0 & 0 & 0 \\
\hline TDB-10 & 9 & 12 & 13 & 25 & 0 & 7 & 15 & 15 & 22 & 7 & 9 & 0 & 22 & 10 & 20 & 12 & 0 & 8 \\
\hline TDG-10 & 7 & 9 & 7 & 9 & 9 & 14 & 12 & 8 & 0 & 0 & 9 & 0 & 0 & 4 & 0 & 0 & 22 & 6 \\
\hline TDJ-10,18 & 0 & 6 & 0 & 0 & 0 & 0 & 0 & 0 & 0 & 0 & 0 & 0 & 0 & 0 & 0 & 0 & 0 & 0 \\
\hline TDR-10 & 0 & 0 & 0 & 0 & 0 & 0 & 0 & 0 & 0 & 0 & 0 & 0 & 0 & 0 & 0 & 0 & 0 & 12 \\
\hline TFB-10 & 12 & 0 & 4 & 3 & 13 & 5 & 4 & 17 & 0 & 9 & 0 & 0 & 0 & 10 & 4 & 3 & 0 & 8 \\
\hline TFG-10 & 0 & 0 & 0 & 0 & 3 & 6 & 0 & 0 & 0 & 3 & 0 & 0 & 0 & 1 & 0 & 0 & 0 & 0 \\
\hline TLG-18 & 0 & 6 & 0 & 1 & 3 & 0 & 0 & 0 & 0 & 1 & 0 & 0 & 0 & 0 & 0 & 0 & 0 & 0 \\
\hline Isolates (no.) & 76 & 34 & 69 & 117 & 67 & 111 & 26 & 59 & 9 & 68 & 23 & 46 & 9 & 73 & 46 & 116 & 9 & 66 \\
\hline
\end{tabular}

y Races based on 12 North American leaf rust differential lines (10) plus supplemental lines with $\operatorname{Lr} 10$ and $\operatorname{Lr} 18$.

${ }^{\mathrm{z}}$ Agroecological areas are shown in Figure 1. 
red winter wheat region (areas 1, 2, and 3), and in TL- races, found mostly in the Southeast (area 1).

Virulence to Lrl6 decreased from $17 \%$ in 1986 in the U.S. population to less than $2 \%$ in 1995. In 1995, four phenotypes (CGB, MGB, MGG, and MJB) were identified with virulence to Lrl6. In Canada,
Table 4. Races of Puccinia triticina collected from fields (F) and nurseries (N) in agroecological areas 7 (Southwest) and 8 (Northwest) of the United States in 1993, 1994, and 1995

\begin{tabular}{|c|c|c|c|c|c|c|c|c|c|c|c|c|}
\hline \multirow[b]{4}{*}{ Code $^{y}$} & \multicolumn{12}{|c|}{ Percentage of isolates from indicated source } \\
\hline & \multicolumn{6}{|c|}{ Area $7^{z}$} & \multicolumn{6}{|c|}{ Area 8} \\
\hline & \multicolumn{2}{|c|}{1993} & \multicolumn{2}{|c|}{1994} & \multicolumn{2}{|c|}{1995} & \multicolumn{2}{|c|}{1993} & \multicolumn{2}{|c|}{1994} & \multicolumn{2}{|c|}{1995} \\
\hline & $\mathbf{F}$ & $\mathbf{N}$ & $\mathbf{F}$ & $\mathbf{N}$ & $\mathbf{F}$ & $\mathbf{N}$ & $\mathbf{F}$ & $\mathbf{N}$ & $\mathbf{F}$ & $\mathbf{N}$ & $\mathbf{F}$ & $\mathbf{N}$ \\
\hline CCB-10 & 8 & 4 & 0 & 0 & 0 & 0 & 0 & 0 & 0 & 0 & 0 & 0 \\
\hline LBG-10 & 0 & 0 & 0 & 0 & 0 & 0 & 0 & 0 & 0 & 29 & 0 & 0 \\
\hline LBG-10,18 & 0 & 0 & 0 & 0 & 0 & 0 & 0 & 0 & 0 & 5 & 0 & 0 \\
\hline MBB-10 & 8 & 32 & 0 & 33 & 53 & 23 & 0 & 0 & 0 & 5 & 0 & 0 \\
\hline MBB-10,18 & 0 & 0 & 0 & 0 & 0 & 0 & 27 & 0 & 0 & 0 & 0 & 0 \\
\hline MBD-10 & 0 & 0 & 0 & 0 & 0 & 0 & 0 & 0 & 0 & 5 & 0 & 0 \\
\hline MBG-18 & 8 & 0 & 0 & 0 & 0 & 0 & 0 & 0 & 0 & 0 & 0 & 0 \\
\hline MBG-10 & 0 & 8 & 25 & 15 & 13 & 38 & 0 & 0 & 0 & 14 & 0 & 0 \\
\hline MBM-10 & 0 & 0 & 0 & 0 & 0 & 0 & 0 & 11 & 0 & 0 & 0 & 0 \\
\hline MBR-10 & 0 & 0 & 0 & 4 & 0 & 3 & 0 & 0 & 0 & 0 & 0 & 0 \\
\hline MCB-10 & 54 & 56 & 75 & 30 & 0 & 13 & 0 & 0 & 0 & 10 & 100 & 0 \\
\hline MCD-10 & 0 & 0 & 0 & 7 & 0 & 0 & 0 & 0 & 0 & 0 & 0 & 0 \\
\hline MCG-10 & 0 & 0 & 0 & 11 & 33 & 25 & 0 & 0 & 0 & 0 & 0 & 55 \\
\hline MCR-10 & 0 & 0 & 0 & 0 & 0 & 0 & 0 & 5 & 0 & 0 & 0 & 0 \\
\hline MDB-10 & 0 & 0 & 0 & 0 & 0 & 0 & 9 & 11 & 0 & 0 & 0 & 18 \\
\hline NBB-10,18 & 0 & 0 & 0 & 0 & 0 & 0 & 27 & 21 & 0 & 29 & 0 & 0 \\
\hline NCB-10 & 0 & 0 & 0 & 0 & 0 & 0 & 9 & 0 & 0 & 0 & 0 & 0 \\
\hline PBD-10 & 0 & 0 & 0 & 0 & 0 & 0 & 18 & 0 & 0 & 0 & 0 & 0 \\
\hline PBG-10 & 0 & 0 & 0 & 0 & 0 & 0 & 0 & 0 & 0 & 5 & 0 & 0 \\
\hline PBJ-10,18 & 0 & 0 & 0 & 0 & 0 & 0 & 0 & 0 & 0 & 0 & 0 & 9 \\
\hline PBP-10 & 0 & 0 & 0 & 0 & 0 & 0 & 0 & 0 & 0 & 0 & 0 & 18 \\
\hline PGD-10 & 0 & 0 & 0 & 0 & 0 & 0 & 9 & 53 & 0 & 0 & 0 & 0 \\
\hline PNM-10,18 & 8 & 0 & 0 & 0 & 0 & 0 & 0 & 0 & 0 & 0 & 0 & 0 \\
\hline TDG-10 & 15 & 0 & 0 & 0 & 0 & 0 & 0 & 0 & 0 & 0 & 0 & 0 \\
\hline Isolates (no.) & 13 & 25 & 8 & 27 & 15 & 40 & 11 & 19 & 0 & 21 & 2 & 11 \\
\hline
\end{tabular}

virulence to $\operatorname{Lrl6}$ occurred in $6 \%$ of the isolates in 1987 (5) and 6\% in 1995 (8), but it was not found from 1989 to 1994.

Virulence to $\operatorname{Lr} 30$ was relatively common in most areas of the U.S. except the Southwest (area 7) and the Pacific Northwest (area 8) from 1993 to 1995.

From the 1993 to 1995 bulked collections, no virulence was found to 12 resistant series entries: Aepoglom, Arapahoe, AZ-FH 50-4-1-1-1, AZ-FH 50-1-1-1-1, Buck Manantial, Ceruga 3, Era/Lr19, KS86WGRC16(Lr43), Thatcher Lr19, TcLr26/TcLr29//TcLr19/3/TcLr26/TcLr21, Tc $L r 29 / \mathrm{Tc} L r 21 / / \mathrm{Tc} L r 25 / 3 /$ Stoa, and Norm.

Diversity and genetic distance. Shannon indexes of diversity of leaf rust races collected from 1993 through 1995 were greatest in area 3 (Ohio Valley) in both field and nursery collections (Fig. 3). Diversity was also high in fields in area 4 (southern Great Plains). For nurseries, Shannon indexes did not differ significantly among areas $2,4,6$, and 8 . In fields, areas $1,5,6$, and 8 had similar Shannon indexes that were significantly less than those for areas 3 and 4. Areas 2 (Northeast) and 7 (Southwest) had the smallest Shannon indexes for diversity of races in the field collections, and area 7 had the smallest Shannon index for the nursery collections. The apparent low diversity of races in areas 2 and 8 should be viewed with caution because of the low number of collections (less than $50 \%$ of the mean number over all areas) obtained from areas 2 and 8 . When sample sizes differ markedly,

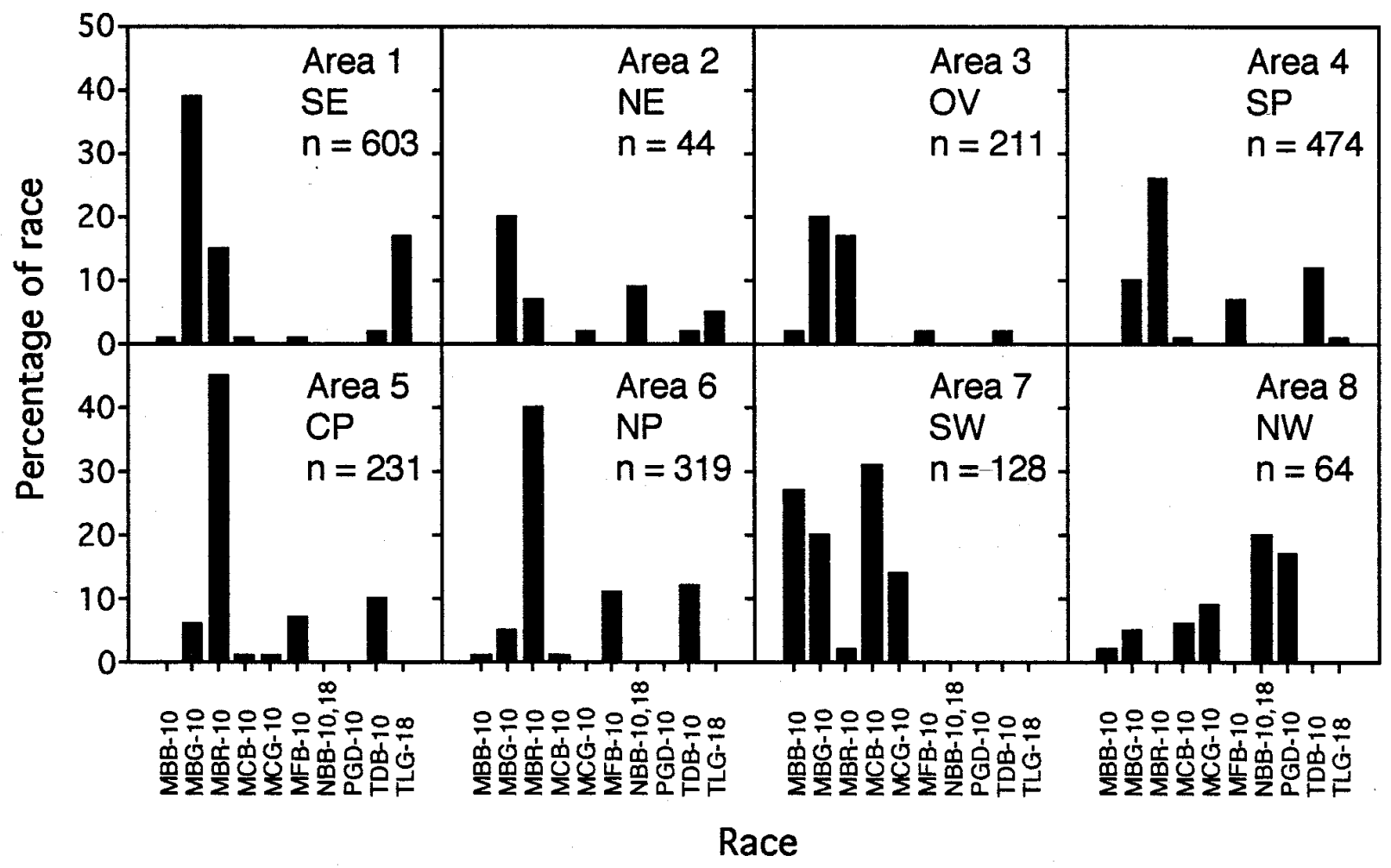

Fig. 2. Frequency of common races (greater than $10 \%$ in at least one area) of Puccinia triticina among collections from areas 1 to 8 of the United States (see Figure 1 for area identification) from 1993 through 1995. Collections from fields and nurseries are combined. 
Shannon index values underestimate diversity within the smaller samples relative to the larger. Similarly, Shannon indexes for field and nursery collections in area 1 may overestimate the diversity in area 1 relative to other areas, because the sample sizes from area 1 were more than twice the mean values for field and nursery samples from all areas. The number of isolates from fields in area 4 also was greater than twice the mean sample size for all field collections, so diversity or races in fields of area 4 may be overestimated by the Shannon index. Taking sample size into account, however, it is clear that in 1993 to 1995 , diversity of races from fields was greater in area 3 than in areas $1,5,6$, or 7 ; and diversity of races from nurseries was greater in area 3 than in areas $1,4,5,6$, or 7.
In areas 2, 6, and 8, Shannon indexes were significantly greater for nursery collections than among field collections (Fig. 3), but this could be due to the much larger numbers of isolates collected from nurseries than from fields in those areas. The greater diversity in fields than in nurseries in area 4 cannot be attributed to differences in sample size, because the number of isolates from fields in area 4 was less than the

Table 5. Percentage of isolates of Puccinia triticina virulent on the single-gene differential lines used in the 1993-1995 surveys

\begin{tabular}{|c|c|c|c|c|c|c|c|c|c|c|c|c|c|c|c|c|c|}
\hline \multirow[b]{2}{*}{ Year } & \multirow[b]{2}{*}{ Area } & \multirow[b]{2}{*}{ Source } & \multirow{2}{*}{$\begin{array}{l}\text { No. of } \\
\text { isolates }\end{array}$} & \multicolumn{14}{|c|}{ Percentage of isolates virulent on $L r$ gene } \\
\hline & & & & 1 & $2 a$ & $2 c$ & 3 & $3 k a$ & 9 & 10 & 11 & 16 & 17 & 18 & 24 & 26 & 30 \\
\hline 1993 & 1 & Field & 74 & 95 & 20 & 43 & 100 & 27 & 35 & 72 & 74 & 0 & 1 & 42 & 20 & 5 & 27 \\
\hline 1994 & 1 & Field & 27 & 100 & 33 & 33 & 96 & 22 & 22 & 85 & 74 & 0 & 0 & 30 & 19 & 0 & 22 \\
\hline 1995 & 1 & Field & 78 & 99 & 24 & 24 & 97 & 27 & 18 & 72 & 100 & 0 & 1 & 31 & 4 & 10 & 27 \\
\hline 1993 & 1 & Nursery & 140 & 97 & 44 & 55 & 98 & 11 & 18 & 60 & 84 & 0 & 3 & 45 & 14 & 4 & 11 \\
\hline 1994 & 1 & Nursery & 125 & 99 & 16 & 19 & 100 & 35 & 6 & 95 & 82 & 0 & 0 & 6 & 16 & 6 & 35 \\
\hline 1995 & 1 & Nursery & 159 & 100 & 18 & 22 & 94 & 24 & 18 & 83 & 94 & 0 & 0 & 30 & 4 & 7 & 24 \\
\hline 1994 & 2 & Field & 6 & 100 & 17 & 100 & 100 & 83 & 50 & 100 & 67 & 0 & 0 & 83 & 0 & 50 & 83 \\
\hline 1995 & 2 & Field & 4 & 100 & 0 & 50 & 100 & 50 & 50 & 100 & 50 & 0 & 0 & 50 & 50 & 0 & 50 \\
\hline 1993 & 2 & Nursery & 10 & 90 & 0 & 80 & 40 & 30 & 0 & 90 & 10 & 0 & 0 & 60 & 0 & 0 & 0 \\
\hline 1994 & 2 & Nursery & 12 & 100 & 17 & 42 & 100 & 50 & 0 & 92 & 83 & 0 & 0 & 25 & 8 & 0 & 50 \\
\hline 1995 & 2 & Nursery & 12 & 100 & 17 & 42 & 67 & 8 & 17 & 83 & 58 & 0 & 0 & 58 & 0 & 17 & 0 \\
\hline 1993 & 3 & Field & 29 & 41 & 3 & 69 & 97 & 69 & 3 & 38 & 31 & 0 & 3 & 45 & 14 & 21 & 69 \\
\hline 1994 & 3 & Field & 18 & 72 & 6 & 22 & 100 & 56 & 11 & 83 & 67 & 0 & 0 & 17 & 28 & 28 & 67 \\
\hline 1995 & 3 & Field & 32 & 97 & 13 & 13 & 100 & 50 & 0 & 94 & 91 & 0 & 0 & 6 & 13 & 34 & 50 \\
\hline 1993 & 3 & Nursery & 82 & 99 & 35 & 38 & 100 & 15 & 1 & 96 & 57 & 2 & 0 & 2 & 39 & 26 & 13 \\
\hline 1994 & 3 & Nursery & 24 & 67 & 17 & 50 & 100 & 63 & 0 & 54 & 67 & 0 & 0 & 33 & 17 & 0 & 63 \\
\hline 1995 & 3 & Nursery & 26 & 81 & 0 & 12 & 100 & 85 & 12 & 85 & 73 & 0 & 0 & 31 & 12 & 38 & 81 \\
\hline 1993 & 4 & Field & 76 & 89 & 39 & 42 & 96 & 11 & 0 & 97 & 39 & 0 & 12 & 1 & 54 & 34 & 11 \\
\hline 1994 & 4 & Field & 69 & 100 & 36 & 38 & 91 & 36 & 0 & 91 & 62 & 3 & 13 & 0 & 38 & 14 & 36 \\
\hline 1995 & 4 & Field & 67 & 100 & 34 & 37 & 94 & 51 & 6 & 91 & 73 & 3 & 9 & 6 & 43 & 24 & 51 \\
\hline 1993 & 4 & Nursery & 34 & 94 & 50 & 56 & 94 & 15 & 12 & 94 & 53 & 0 & 9 & 18 & 38 & 15 & 15 \\
\hline 1994 & 4 & Nursery & 117 & 99 & 41 & 41 & 100 & 32 & 1 & 99 & 51 & 0 & 3 & 1 & 55 & 17 & 32 \\
\hline 1995 & 4 & Nursery & 111 & 97 & 36 & 39 & 100 & 44 & 0 & 98 & 76 & 3 & 5 & 0 & 50 & 20 & 44 \\
\hline 1993 & 5 & Field & 26 & 88 & 50 & 50 & 100 & 4 & 0 & 96 & 54 & 0 & 0 & 0 & 50 & 38 & 4 \\
\hline 1994 & 5 & Field & 9 & 100 & 22 & 22 & 100 & 78 & 0 & 100 & 78 & 0 & 0 & 0 & 22 & 11 & 78 \\
\hline 1995 & 5 & Field & 23 & 100 & 22 & 22 & 100 & 65 & 0 & 100 & 91 & 0 & 0 & 0 & 17 & 0 & 65 \\
\hline 1993 & 5 & Nursery & 59 & 93 & 54 & 54 & 100 & 25 & 0 & 98 & 37 & 0 & 3 & 0 & 68 & 42 & 25 \\
\hline 1994 & 5 & Nursery & 68 & 100 & 25 & 25 & 100 & 63 & 1 & 99 & 78 & 0 & 3 & 1 & 22 & 15 & 63 \\
\hline 1995 & 5 & Nursery & 46 & 96 & 0 & 0 & 100 & 70 & 0 & 100 & 85 & 11 & 0 & 0 & 24 & 4 & 70 \\
\hline 1993 & 6 & Field & 9 & 100 & 33 & 33 & 100 & 22 & 0 & 100 & 33 & 0 & 11 & 0 & 56 & 33 & 22 \\
\hline 1994 & 6 & Field & 46 & 100 & 26 & 26 & 100 & 54 & 0 & 100 & 65 & 0 & 0 & 0 & 39 & 15 & 54 \\
\hline 1995 & 6 & Field & 9 & 100 & 56 & 56 & 100 & 33 & 0 & 100 & 78 & 0 & 0 & 0 & 56 & 0 & 33 \\
\hline 1993 & 6 & Nursery & 73 & 89 & 41 & 42 & 96 & 16 & 1 & 100 & 37 & 0 & 7 & 1 & 56 & 42 & 16 \\
\hline 1994 & 6 & Nursery & 116 & 100 & 18 & 19 & 100 & 61 & 0 & 100 & 69 & 0 & 1 & 1 & 35 & 18 & 61 \\
\hline 1995 & 6 & Nursery & 66 & 100 & 36 & 36 & 100 & 65 & 0 & 100 & 74 & 3 & 0 & 0 & 47 & 12 & 65 \\
\hline 1993 & 7 & Field & 13 & 92 & 15 & 23 & 100 & 8 & 8 & 92 & 23 & 0 & 0 & 15 & 23 & 62 & 8 \\
\hline 1994 & 7 & Field & 8 & 100 & 0 & 0 & 100 & 0 & 0 & 100 & 25 & 0 & 0 & 0 & 0 & 75 & 0 \\
\hline 1995 & 7 & Field & 15 & 100 & 0 & 0 & 100 & 0 & 0 & 100 & 47 & 0 & 0 & 0 & 0 & 33 & 0 \\
\hline 1993 & 7 & Nursery & 25 & 96 & 0 & 0 & 100 & 0 & 0 & 100 & 8 & 0 & 0 & 0 & 0 & 60 & 0 \\
\hline 1994 & 7 & Nursery & 27 & 100 & 0 & 0 & 100 & 4 & 0 & 100 & 30 & 0 & 7 & 0 & 0 & 48 & 4 \\
\hline 1995 & 7 & Nursery & 40 & 100 & 0 & 0 & 100 & 3 & 0 & 100 & 65 & 0 & 0 & 0 & 0 & 38 & 3 \\
\hline 1993 & 8 & Field & 11 & 100 & 0 & 27 & 100 & 0 & 0 & 100 & 0 & 9 & 27 & 55 & 9 & 9 & 0 \\
\hline 1995 & 8 & Field & 2 & 100 & 0 & 0 & 100 & 0 & 0 & 100 & 0 & 0 & 0 & 0 & 0 & 100 & 0 \\
\hline 1993 & 8 & Nursery & 19 & 100 & 0 & 74 & 79 & 16 & 0 & 100 & 5 & 53 & 53 & 21 & 11 & 5 & 16 \\
\hline 1994 & 8 & Nursey & 21 & 100 & 0 & 33 & 38 & 0 & 0 & 100 & 52 & 0 & 5 & 33 & 0 & 10 & 0 \\
\hline 1995 & 8 & Nursery & 11 & 100 & 0 & 27 & 100 & 18 & 0 & 100 & 64 & 0 & 27 & 9 & 18 & 55 & 18 \\
\hline \multicolumn{18}{|l|}{ Totals } \\
\hline 1993 & U.S. & Field & 238 & 86 & 27 & 45 & 98 & 22 & 12 & 82 & 48 & 0 & 4 & 22 & 34 & 24 & 22 \\
\hline 1994 & U.S. & Field & 183 & 97 & 27 & 32 & 96 & 43 & 6 & 93 & 64 & 1 & 5 & 9 & 31 & 17 & 44 \\
\hline 1995 & U.S. & Field & 230 & 99 & 24 & 26 & 97 & 40 & 9 & 87 & 84 & 1 & 3 & 14 & 20 & 18 & 40 \\
\hline 1993 & U.S. & Nursery & 442 & 95 & 38 & 48 & 96 & 15 & 7 & 86 & 53 & 3 & 5 & 19 & 33 & 24 & 14 \\
\hline 1994 & U.S. & Nursery & 510 & 98 & 22 & 26 & 97 & 43 & 2 & 96 & 67 & 0 & 2 & 6 & 28 & 15 & 43 \\
\hline 1995 & U.S. & Nursery & 471 & 98 & 20 & 24 & 97 & 40 & 7 & 93 & 81 & 2 & 2 & 13 & 23 & 16 & 39 \\
\hline \multicolumn{18}{|c|}{ Totals - field and nursery } \\
\hline 1993 & U.S. & & 680 & 92 & 34 & 47 & 97 & 17 & 9 & 84 & 51 & 2 & 5 & 20 & 34 & 24 & 17 \\
\hline 1994 & U.S. & & 693 & 98 & 23 & 28 & 97 & 43 & 3 & 95 & 66 & 0 & 3 & 6 & 29 & 15 & 43 \\
\hline 1995 & U.S. & & 701 & 98 & 22 & 25 & 97 & 40 & 8 & 91 & 82 & 2 & 2 & 14 & 22 & 17 & 40 \\
\hline \multicolumn{18}{|l|}{ Total } \\
\hline 1993-95 & U.S. & & 2,074 & 96 & 26 & 33 & 97 & 33 & 6 & 90 & 67 & 1 & 3 & 13 & 28 & 19 & 33 \\
\hline
\end{tabular}


number from nurseries. In past surveys, the diversity of races has generally been greater from nurseries than from fields in area 2 , even though sample sizes did not differ greatly between area 2 fields and nurseries $(9,12,13)$. There have not been consistent differences in diversity between field and nursery collections in other areas analyzed from 1988 to 1995 .

In general, the levels of diversity of races found in areas 1 to 8 in 1993 to 1995 were similar to those found in earlier analyses for surveys from 1988 to 1992 $(9,12,13)$. This indicates that, with exceptions noted below, the number of races and the relative dominance of races found in each area did not differ greatly from year to year. The following differences were noted: In 1991, when very few isolates were collected from fields in area 1 , the Shannon index of 0.7 for area 1 field collections was much lower than for other surveys in which the Shannon index was similar to the value of 2.2 for 1993 to 1995. Since 1991, the numbers of collections from fields in area 2 have been relatively low, and the Shannon indexes of diversity (1.62, 2.03, and 2.19 for 1991, 1992 , and 1993 to 1995 ) also have been much lower than the value 2.7 found in 1988 to 1990 . Field collections from area 3 usually exhibit the greatest diversity of races, as they did in 1993 to 1995 , but in the 1992 survey, the Shannon index for area 3 was only 1.9 , whereas it was 2.7 in 1993 to 1995 . In 1992, however, the number of collections from fields in area 3 was lower than that of all other areas except 7 and 8. Diversity in field collections from area 4 was high in $1992\left(\mathrm{H}_{\mathrm{w}}=2.6\right)$ as well as in 1993 to $1995\left(\mathrm{H}_{\mathrm{w}}=2.7\right)$ compared with earlier years that had similar sample sizes $\left(H_{w}=2.2\right.$ for 1988 to 1990 and 2.1 for 1991). The generally high diversity of races in area 3 fields may reflect migration of $P$. triticina spores into area 3 from both area 1 and area 4.

For nursery collections, the Shannon indexes for areas 1 and 2 (2.8 and 3.2, respectively) were much higher in 1988 to 1990 than for subsequent surveys. This may be attributed to larger sample size for area 2 in 1988 to 1990 , but not for area 1 , in which samples sizes were greater in 1991 to 1995 than in 1988 to 1990 . The Shannon index of 2.8 for nursery collections from area 3 in 1993 to 1995 was much greater than the values 2.1 and 2.0 for 1991 and 1992, when sample sizes were somewhat smaller. Also, the diversity of races among collections from nurseries in area 8 was much greater in 1993 to 1995 $\left(\mathrm{H}_{\mathrm{w}}=2.4\right)$ than in previous surveys, in which the Shannon index was less than 2.0 ; this is probably due to the low sample sizes for nurseries in area 8 from 1988 to 1992.

As in previous surveys $(9,12,13)$, Rogers' indexes for genetic distances between leaf rust collections from areas 4, 5, and 6 in the Great Plains were low, indicating that occurrence and frequency of leaf rust races were similar throughout the Great Plains, as expected for a single epidemiological unit (Fig. 4A). Leaf rust overwinters every year in area 4 and parts of area 5 and spreads north with the developing wheat crop. Since 1988, the Rogers' indexes for differences among field collections from areas 4,5 , and 6 have been in

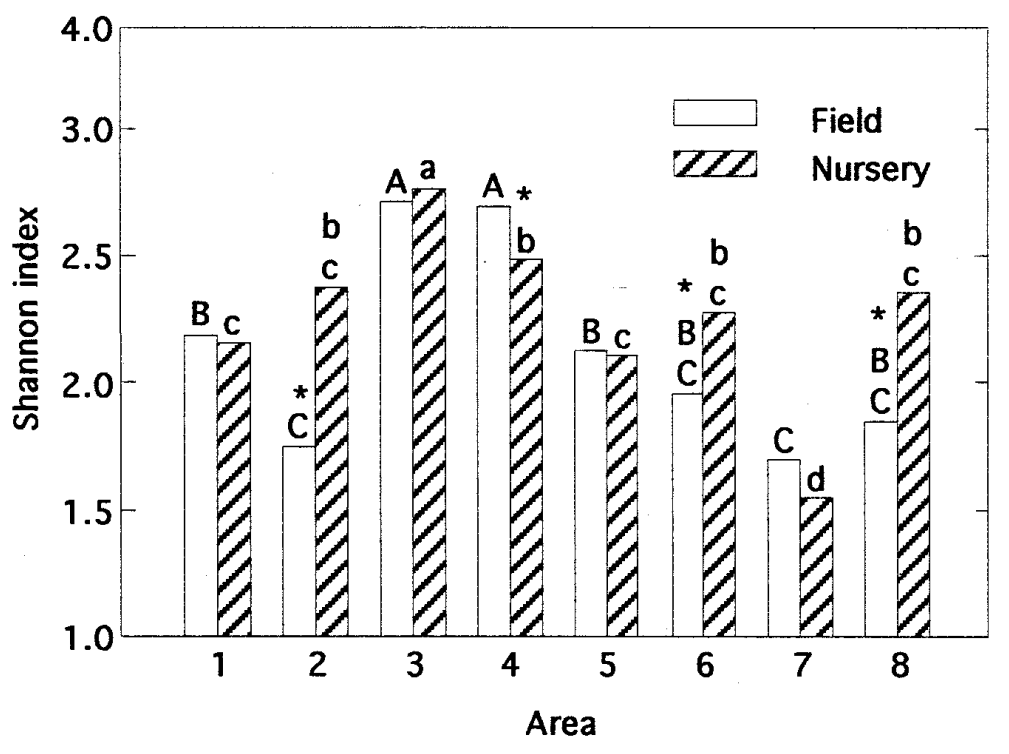

Fig. 3. Comparison of Shannon indexes of diversity in field and nursery collections of Puccinia triticina from areas 1 to 8 in the United States from 1993 through 1995. Diversity indexes that do not differ significantly $(P>0.05)$ are indicated by identical letter (uppercase for field collections, lowercase for nursery collections). Significant differences in diversity between field and nursery collections within an area are indicated by asterisks.

the range of 0.3 to 0.4 . Field collections from areas 1 and 3 showed a low level of similarity (Rogers' index near 0.6) in 1992 and in the period 1993 to 1995 . No apparent association between areas 1 and 3 was seen in earlier years. Field collections in areas 2, 7 , and 8 typically have shown little similarity to each other or to collections from other areas. This was true again in 1993 to 1995.

As with the field collections of leaf rust, areas 4,5 , and 6 were quite similar in occurrence and frequency of races collected from nurseries in 1993 to 1995 (Fig. 4B) or earlier years. Rogers' indexes for areas 4 , 5 , and 6 have been in the range of 0.3 to 0.4 since $1988(9,12,13)$. Nursery collections from areas 2,7 , and 8 showed little similarity with each other or with the other areas in 1993 to 1995 or in earlier surveys. Unlike the field collections, there has been a consistent similarity of nursery collections from areas 1 and 3 over the years from 1988 to 1995. Rogers' indexes have been in the range of 0.3 to 0.4 for most years, although in 1993 to 1995, the Rogers' index for genetic distance between nursery collections in areas 1 and 3 was higher, at 0.48 .

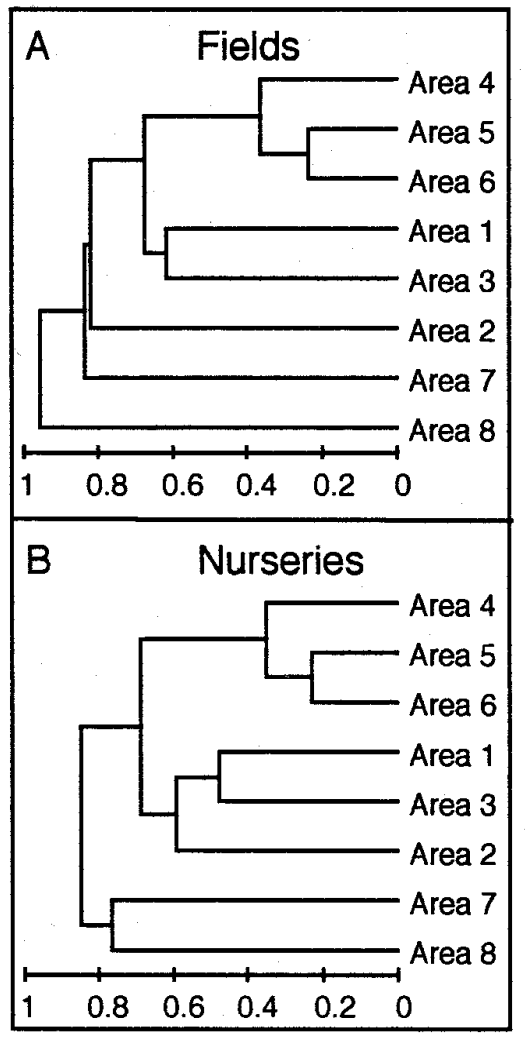

Fig. 4. Phenogram of similarities of racial compositions based on Rogers' index for collections of Puccinia triticina isolates in 1993 to 1995 from (A) fields and (B) nurseries in areas 1 to 8 (Area $1=$ Southeast, Area $2=$ Northeast, Area 3 = Ohio Valley, Area $4=$ Southern Plains, Area $5=$ Central Plains, Area $6=$ Northern Plains, Area $7=$ Southwest, and Area $8=$ Northwest). 
The similarity index comparisons showed that collections from both fields and nurseries of areas 4, 5, and 6 had very similar virulence frequencies (i.e., low SI values) (Figs. 5A and B). Similar patterns were seen with Nei's distances calculated in previous surveys $(9,12,13)$. Also, Nei's distance calculations in earlier surveys indicated that collections from both fields and nurseries in areas 1 and 3 had similar virulence frequencies in most years. Similarity indexes for the 1993 to 1995 field data also revealed that virulence frequencies of leaf rust populations in areas 1 and 3 were more similar to each other than to those in other areas. For nursery collections, however, the virulence frequencies in 1993 to 1995 for area 3 were more similar to those for areas 4, 5, and 6 than to virulence frequencies for area 1. From 1988 to 1992, nursery collections from area 2 have had virulence frequencies relatively similar to those in area 8. By the new similarity index, virulence frequencies in nurseries of area 2 in 1993 to 1995 showed greater similarity to those in area 1 than in area 8 . Surveys before 1993 did not include sufficient numbers of isolates from fields in

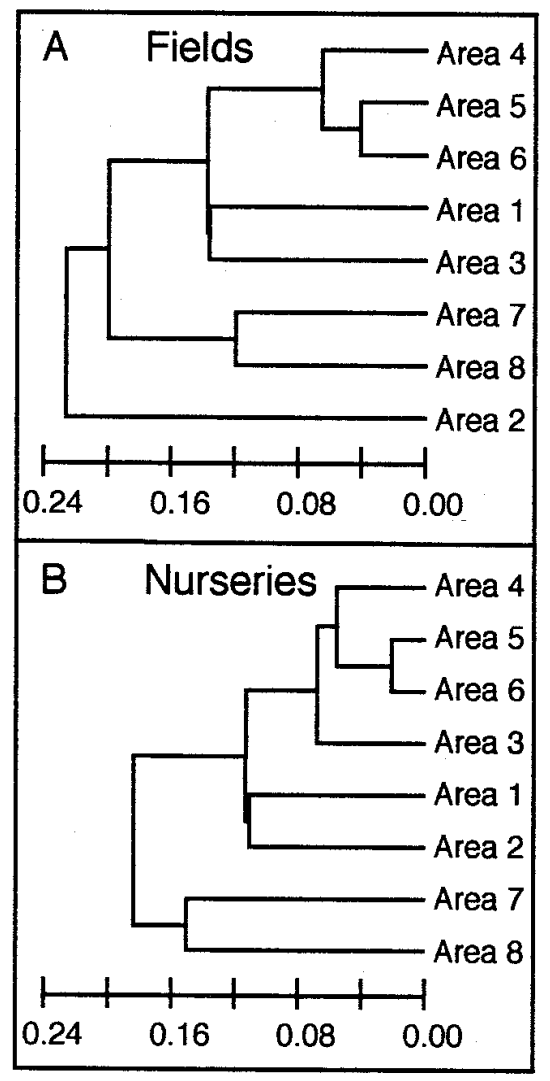

Fig. 5. Phenogram of similarity indexes of virulence based on mean absolute value of differences in virulence frequencies between collections of Puccinia triticina in 1993 to 1995 from (A) fields and (B) nurseries in areas 1 to 8 (Area $1=$ Southeast, Area $2=$ Northeast, Area 3 = Ohio Valley, Area $4=$ Southern Plains, Area $5=$ Central Plains, Area $6=$ Northern Plains, Area $7=$ Southwest, and Area $8=$ Northwest). area 8 for a comparison between virulence frequencies in area 8 and those in other areas. The 1993 to 1995 surveys, however, showed considerable similarity of virulence frequencies in field collections from areas 7 and 8 but less similarity of virulence frequencies in nursery collections from areas 7 and 8 .

In addition to calculating the new similarity indexes, we also calculated the adapted Nei's distances used in previous surveys in order to compare the two ways of assessing similarities in virulence frequencies between populations. In general, the phenograms developed with the adapted Nei's distance (data not shown) were qualitatively similar to those shown in Figure 5 for the new similarity index. The only exception was in the phenogram for nursery collections: Nei's distance indicated a closer relationship of area 2 to area 8 ; whereas the similarity index showed more similarity between areas 2 and 1 than between 2 and 8 .

Over all areas, the Nei's distances were less in 1993 to 1995 than in previous surveys. For field collections, the greatest Nei's distance between area collections ranged from 0.19 to 0.25 for 1988 to 1992 compared with 0.14 for 1993 to 1995 , and for nursery collections, the greatest Nei's distances ranged from 0.10 to 0.27 for 1988 to 1992 compared with 0.07 for 1993 to 1995 . This indicates a reduction in regional diversity of virulence frequencies in recent years. On the other hand, the Rogers' distances have not shown a similar decrease over all regions in recent years. Therefore, it appears that even though virulence frequencies have become somewhat more similar among areas, the specific races that make up the area populations are still relatively distinct except in the single epidemiological unit comprised of areas 4, 5, and 6 .

In addition to calculating genetic distances among areas, we also calculated the Rogers' index for differences in occurrence and frequency of races between field and nursery collections within areas. Within areas 1, 6, and 7, Rogers' indexes for differences in occurrence and frequency of races in field collections compared with nursery collections in 1993 to 1995 were $0.19,0.19$, and 0.20 , respectively. These values are lower than those for differences between areas 5 and 6 , which are in the same epidemiological unit. Field collections compared with nursery collections within areas 3, 4, and 5 had Rogers' indexes of $0.47,0.29$, and 0.34 , which are within the same range as the Rogers' indexes comparing area 4 with areas 5 and 6 within the same epidemiological unit.

On the other hand, nursery collections were very different from field collections in areas 2 and 8, where Rogers' indexes were 0.71 and 0.61 , respectively. In 1991 and 1992, the Rogers' indexes for field and nursery collections in area 2 were even greater, at 0.85 and 0.82 , respectively $(12,13)$. Values this high indicate almost no relationship between the leaf rust races found in fields and those from nurseries. We did not calculate Rogers' indexes between field and nursery collections in area 8 in 1991 and 1992, because there were no field collections from area 8 in those years. As indicated previously $(12,13)$, high Rogers' indexes for differences between field and nursery collections within area 2 suggest that area 2 may comprise multiple epidemiological units. This could result from local survival of leaf rust throughout the year and from geographical features such as large discontinuities in local areas of wheat production and mountain ranges that restrict long-distance movement of rust spores (24).

\section{ACKNOWLEDGMENTS}

We thank Mark Hughes and Thomas Schibillia for data analysis and technical assistance.

\section{LITERATURE CITED}

1. Anikster, Y., Bushnell, W. R., Eilam, T. Manisterski, J., and Roelfs, A. P. 1997. Puccinia recondita causing leaf rust on cultivated wheats, wild wheats, and rye. Can. J. Bot. 75:2082-2096.

2. Cox, T. S., Raupp, W. J., and Gill, B. S. 1994. Leaf rust-resistance genes $L r 41, L r 42$, and Lr43 transferred from Triticum tauschii to common wheat. Crop Sci. 34:339-343.

3. Dyck, P. L. 1989. The inheritance of leaf rust resistance in wheat cultivars Kenyon and Buck Manantial. Can. J. Plant Sci. 69:11131117.

4. Groth, J. V., and Roelfs, A. P. 1987. The concept and measurement of phenotypic diversity in Puccinia graminis on wheat. Phytopathology 77:1395-1399.

5. Kolmer, J. A. 1989. Virulence and race dynamics of Puccinia recondita $\mathrm{f}$. sp. tritici in Canada during 1956-1987. Phytopathology 79:349-356.

6. Kolmer, J. A. 1991. Phenotypic diversity in two populations of Puccinia recondita f. sp. tritici in Canada during 1931-1987. Phytopathology 81:311-315.

7. Kolmer, J. A. 1992. Diversity of virulence phenotypes and effect of host sampling between and within populations of Puccinia recondita f. sp. tritici in Canada. Plant Dis. 76:618-621.

8. Kolmer, J. A., and Liu, J. Q. 1997. Physiologic specialization of Puccinia recondita $\mathrm{f}$ sp. tritici in Canada in 1995. Can. J. Plant Pathol. 19:166-170

9. Leonard, K. J., Roelfs, A. P., and Long, D. L. 1992. Diversity of virulence within and among populations of Puccinia recondita $\mathrm{f}$. sp. tritici in different areas of the United States. Plant Dis. 76:500-504.

10. Long, D. L., and Kolmer, J. A. 1989. A North American system of nomenclature for Puccinia recondita f. sp. tritici. Phytopathology 79:525-529.

11. Long, D. L., Roberts, J. J., Schafer, J. F. Johnson, J. W., Fowler, H. A., Jr., and Cunfer, B. M. 1992. Registration of six leaf rust resistant soft red winter wheat germplasm lines. Crop Sci. 32:1514-1515.

12. Long, D. L., Roelfs, A. P., Leonard, K. J., and Roberts, J. J. 1993. Virulence and diversity of Puccinia recondita f. sp. tritici in the United States in 1991. Plant Dis. 77:786-791.

13. Long, D. L., Roelfs, A. P., Leonard, K. J., and Roberts, J. J. 1994. Virulence and diversity of Puccinia recondita f. sp. tritici in the United 
States in 1992. Plant Dis. 78:901-906.

14. Long, D. L., Roelfs, A. P., and Roberts, J. J. 1992. Virulence of Puccinia recondita f. sp. tritici in the United States during 1988-1990. Plant Dis. 76:495-499.

15. Long, D. L., Schafer, J. F., and Roelfs, A. P. 1985. Specific virulence of Puccinia recondita f. sp. tritici in the United States from 1978 through 1983. Plant Dis. 69:343-347.

16. Long, D. L., Schafer, J. F., Roelfs, A. P., and Roberts, J. J. 1986. Virulence and epidemiology of Puccinia recondita f. sp. tritici in the United States in 1985. Plant Dis. 70:11071110.

17. Long, D. L., Schafer, J. F., Roelfs, A. P., and Roberts, J. J. 1988. Virulence of Puccinia re- condita f. sp. tritici in the United States in 1986. Plant Dis. 72:22-24.

18. Long, D. L., Schafer, J. F., Roelfs, A. P., and Roberts, J. J. 1989. Virulence of Puccinia recondita f. sp. tritici in the United States in 1987. Plant Dis. 73:294-297.

19. Marshall, D. 1992. Virulence of Puccinia recondita in Texas from 1988 to 1990. Plant Dis. 76:296-299.

20. Nei, M. 1987. Molecular Evolutionary Genetics. Columbia University Press, New York.

21. Poole, R. W. 1974. An Introduction to Quantitative Ecology. McGraw-Hill, New York.

22. Samborski, D. J. 1984. Occurrence and virulence of Puccinia recondita in Canada in 1983. Can. J. Plant Pathol. 6:238-242.
23. Samborski, D. J., and Dyck, P. L. 1982. Enhancement of resistance to Puccinia recondita by interactions of resistance genes in wheat. Can. J. Plant Pathol. 4:152-156.

24. Schafer, J. F., and Long, D. L. 1986. Evidence for local source of Puccinia recondita on wheat in Pennsylvania. Plant Dis. 70:892.

25. Schafer, J. F., and Long, D. L. 1988. Relations of races and virulences of Puccinia recondita f. sp. tritici to wheat cultivars and areas. Plant Dis. 72:25-27.

26. Singh, R. P. 1991. Pathogenicity variations of Puccinia recondita f. $\mathrm{sp}$. tritici and $P$. graminis f. sp. tritici in wheat-growing areas of Mexico during 1988 and 1989. Plant Dis. 75:790-794. 